(20\%), polyarthritis $(15 \%)$, haematological disturbances $(35 \%)$ and persistent immunological markers (30\%). One patient was excluded from the study due to infusion- related flush and severe hypotension reaction while the rest $25 \mathrm{com}$ pleted the observation. We investigated and analyzed haematological results, ANA, anti - ds DNA, anti - Sm autoantibodies and complement fractions at baseline as well on the 12th and 24th month of treatment. The mean SELENA - SLEDAI index changed from 8.9 to 4.5 after 12 months of treatment and remained unchanged until the end of the study. We observed a mean reduction of GCs daily dosage from $12 \mathrm{mg}$ to $6 \mathrm{mg}$ after 18 months.

Conclusion: Biological treatment with Belimumab added to the standard treatment with GCs and AZA in patients with non-lupus nephritis SLE patients adds additional benefits. Our results show that there is a reduction in SLEDAI index as well as reduction in daily GCs intake. Safety profile is not different from the one reported previously.

Disclosure of Interests: None declared

DOI: 10.1136/annrheumdis-2020-eular.2368

\section{AB0369 EFFICACY AND SAFETY OF RITUXIMAB ORIGINATOR AND BIOSIMILAR IN PRIMARY SJÖGREN'S SYNDROME IN A REAL-LIFE SETTING}

F. Carubbi ${ }^{1}$, A. Alunno ${ }^{2}$, P. Cipriani ${ }^{1}$, V. Pavlych ${ }^{1}$, C. DI Muzio ${ }^{1}$, R. Gerli ${ }^{2}$ R. Giacomelli ${ }^{1} .{ }^{1}$ Rheumatology Unit, L'Aquila, Italy; ${ }^{2}$ Rheumatology Unit, Perugia, Italy

Background: Over the last 2 decades rituximab (RTX) has been widely used, albeit off-label, in primary Sjögren's syndrome (pSS). Several studies reported that B-lymphocyte depletion with RTX is effective in this disease not only by reducing disease activity but also by affecting the inflammation and the lymphoid organization that occur in target tissues. With the recent release of several RTX biosimilars (bRTX) on the market, the demonstration of their interchangeability with RTX originator (oRTX) is required.

Objectives: To compare efficacy and safety of oRTX and bRTX in pSS patients in a real-life setting.

Methods: Clinical records of pSS patients referring to a tertiary rheumatology clinic were retrospectively evaluated. Patients having received at least 2 courses of either oRTX or bRTX (1000 mg IV infusion, repeated after 2 weeks - 1 courseand the course repeated after 24 weeks) with complete data at baseline and after 3, 6, 9 and 12 months of treatment were enrolled. Disease activity was assessed with the EULAR SS disease activity index (ESSDAI) and its clinical version without the biological domain (ClinESSDAI). Patient-reported symptoms were assessed with the EULAR SS Patient Reported Index (ESSPRI).

Results: Seven patients that received oRTX and 7 patients that received bRTX were enrolled. Baseline clinical features, including ESSDAI and ESSPRI were similar in the 2 treatment groups. Both compounds significantly reduced ESSDAI and ESSPRI as early as 3 months and no difference between the groups was observed at any time point (Figure 1). Of interest, ESSDAI slowly decreased until month 6 when the most pronounced reduction was observed. Conversely, ESSPRI dropped to its lowest values already at month 3 . With regard to safety, at 12 months of follow-up no adverse event was observed in any of the treatment groups.

Conclusion: At 12 months of follow-up, oRTX and bRTX display similar efficacy and safety profiles. The improvement of patient reported outcomes is faster than the improvement of disease activity with both compounds. Our data support interchangeability of oRTX and bRTX in pSS.

References:

[1] Carubbi F et al. Arthritis Res Ther. 2013;15(5):R172

[2] Carubbi F et al. Lupus. 2014;23(13):1337-49
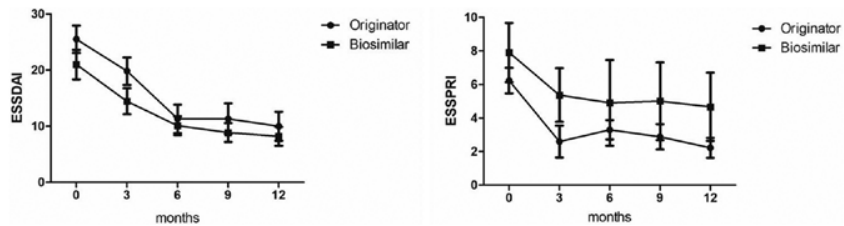

Figure 1 ESSDAI and ESSPRI values at every time point in the 2 treatment groups. Asterisks indicate $p$ values $<0.05$ compared to the other treatment group at the same time point

Disclosure of Interests: Francesco Carubbi Speakers bureau: Francesco Carubbi received speaker honoraria from Abbvie and Celgene outside this work., Alessia Alunno: None declared, Paola Cipriani Grant/research support from: Actelion, Pfizer, Speakers bureau: Actelion, Pfizer, Viktoriya Pavlych: None declared, claudia di muzio: None declared, Roberto Gerli: None declared, Roberto Giacomelli Grant/research support from: Actelion, Pfizer, Speakers bureau: Abbvie, Roche, Actelion, BMS, MSD, Ely Lilly, SOBI, Pfizer DOI: 10.1136/annrheumdis-2020-eular.6608

\begin{tabular}{l|l}
\hline AB0370 & SAFETY OF CS20AT04, A HAPLOIDENTICAL \\
ALLOGENEIC BONE MARROW-DERIVED \\
MESENCHYMAL STEM CELLS, IN A PHASE 1 STUDY \\
IN LUPUS NEPHRITIS
\end{tabular}

C. B. Choi ${ }^{1}$, T. Y. Lee ${ }^{2}$, K. S. Kim², S. C. Bae ${ }^{1}{ }^{1}$ Hanyang University Hospital for Rheumatic Diseases, Rheumatology, Seoul, Korea, Rep. of (South Korea); ${ }^{2}$ Corestem Inc, Seongnam, Korea, Rep. of (South Korea)

Background: Mesenchymal stem cells are known to have immunomodulatory properties and may potentially have therapeutic effect in lupus nephritis. Mesenchymal stem cells form a haploidentical donor are an attractive cell source Objectives: CS20AT04, a haploidentical allogeneic bone marrow-derived mesenchymal stem cell, was evaluated in patients with lupus nephritis for safety and tolerability.

Methods: This was a single-arm phase 1 dose-escalation trial of CS20AT04 in adult patients with lupus nephritis (NCT03174587). A 3+3 design was used for dose escalation. The starting dose was $2.0 \times 106$ cells $/ \mathrm{kg}$ and was escalated to $3.0 \times 106 \mathrm{cell} / \mathrm{kg}$ if there no dose-limiting toxicity. The primary objective was to determine the maximum tolerated dose and evaluate the safety and tolerability at 28 days after the infusion.

Results: Seven patients were enrolled in the study. Patients received CS20AT04 through intravenous infusion. The initial dose of $2.0 \times 106$ cells $/ \mathrm{kg}$ was administered for the first 3 patients without any dose limiting toxicity. There was 1 patient who were not administered the full $2.0 \times 106$ cells $/ \mathrm{kg}$ dose due to technical error during infusion. The patient did not show dose limiting toxicity, but 1 additional patient was enrolled to have 3 patients who received the full $2.0 \times 106$ cells $/ \mathrm{kg}$ dose before escalating to the next level dose. The dose of $3.0 \times 106$ cells $/ \mathrm{kg}$ was administered for the next 3 patients without any dose limiting toxicity. Three adverse events were reported (1 diarrhea, 1 toothache, and 1 arthralgia) and they were all $\mathrm{NCI}-\mathrm{CTC}$ grade I events.

Conclusion: CS20AT04 was well tolerated in single dose up to $3.0 \times 106$ cells/ $\mathrm{kg}$ in patients with lupus nephritis.

Acknowledgments: This research was supported by a grant of the Korea Health Technology R\&D Project through the Korea Health Industry Development Institute (KHIDI), funded by the Ministry of Health \& Welfare, Republic of Korea (grant number: HI15C0778)

Disclosure of Interests: Chan-Bum Choi: None declared, Tae Yong Lee Shareholder of: Corestem Inc, Employee of: Corestem Inc, Kyung Suk Kim Shareholde of: Corestem Inc, Employee of: Corestem Inc, Sang-Cheol Bae: None declared DOI: 10.1136/annrheumdis-2020-eular.3287

\section{$\mathrm{AB} 0371$ \\ HYDROXYCHLOROQUINE AS VIEWED BY LUPUS PATIENTS - WHAT IMPACT FOR DOCTORS?}

A. Cornet ${ }^{1}$, Z. Osmani ${ }^{1,2}$, S. Frankel ${ }^{1}$ on behalf of LUPUS EUROPE PATIENT ADVISORY NETWORK. ${ }^{1}$ Lupus Europe, Romford, United Kingdom; ${ }^{2}$ NVLE, Amsterdam, Netherlands

Background: Hydroxychloroquine ( $\mathrm{HCQ}$ ) is recommended for all patients with systemic lupus erythematosus (SLE) and is typically considered as having a good safety profile ${ }^{1}$. Yet, patient organisations observe that concerns about eye impact or "allergies to HCQ" are often raised on social media. This could contribute to the non-adherence, which varies from 3 to $76 \%$ in SLE patients depending on assessment method and drug $^{2}$.

Objectives: To understand if/how some patients' beliefs impact adherence to HCQ treatment.

Methods: In May 2019, LUPUS EUROPE launched a 29 questions on-line survey in 13 languages including questions on HCQ adherence.

2938 responses were analysed. 67.8\% (1990 patients) were current HCQ users, $17.8 \%$ had stopped using it, $8.1 \%$ never had $\mathrm{HCQ}(6.4 \%$ did not respond to this question). 1820 users reported their adherence level. 314 $(17.3 \%)$ were classified as "low" adherence as they reported missing/forgetting HCQ "always" (1.8\%), "more than twice a week" $(5.2 \%)$ or "once a week" (10.3\%)

Results: The prescribed HCQ dose, kidney involvement or duration of treatment (beyond the $1^{\text {st }}$ year) were found to have no impact on low adherence. Similarly, the user belief that $\mathrm{HCQ}$ has significant side effects, without experiencing these was not found to impact adherence $(p=.74)$.

The following factors were associated with better adherence: $(p<.0001)$ 